\title{
An accurate spline polynomial cubature formula for double integration with logarithmic singularity
}

\begin{abstract}
The paper studied the integration of logarithmic singularity problem $J(\bar{y})=\iint c r(\bar{y}) \log \mid \bar{y}-\bar{y}$ $0 * \mid \mathrm{dA}$, where $\overline{\mathrm{y}}=(\mathrm{U}, \bar{b}), \mathrm{y} 0=(\breve{\mathrm{O}}, \mathrm{b0})$ the domain $q$ is rectangle $q \mathrm{p}=[\mathrm{r} 1, \mathrm{r} 2] \times[\mathrm{r} 3, \mathrm{r} 4]$, the arbitrary point $\bar{y} \in$ opand the fixed point $\bar{y} 0 \in q$ The given density function $G(\bar{y})$, is smooth on

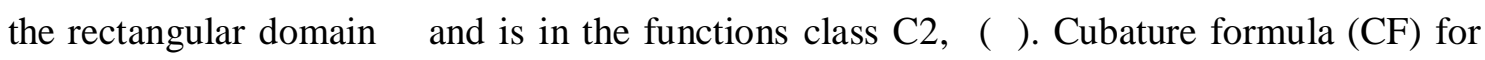
double integration with logarithmic singularities (LS) on a rectangle opis constructed by applying type $(0,2)$ modified spline function $\mathrm{Du}(\mathrm{P})$. The results obtained by testing the density functions $6(\bar{y})$ as linear and absolute value functions shows that the constructed $C F$ is highly accurate.
\end{abstract}

Keyword: Logarithmic singularity; Cubature formula 